\title{
CLAVE TAXONOMICA, ACTUALIZADA, ILUSTRADA Y COMENTADA DE LOS PECES DE LA FAMILIA LABRISOMIDAE DE CHILE (PERCIFORMES, BLENNIOIDEI)
}

\section{UPDATED, ILLUSTRATED AND ANNOTATED TAXONOMIC KEY FOR FISHES OF THE FAMILY LABRISOMIDAE FROM CHILE (PERCIFORMES, BLENNIOIDEI)}

\author{
Sylvia Sáez ${ }^{1}, \&$ Germán Pequeño ${ }^{2}$ \\ 1,2 Instituto de Zoología "Ernst F. Kilian" \\ Universidad Austral de Chile \\ Casilla 567, Valdivia, Chile \\ $\mathrm{c}-\mathrm{e}^{1}=$
}

\begin{abstract}
RESUMEN
Se presenta una clave taxonómica para los integrantes de la familia Labrisomidae de Chile. Dicho taxón está conformado actualmente por Auchenionchus crinitus (Jenyns, 1842), A. microcirrhis (Valenciennes, 1836), A. variolosus (Valenciennes, 1836), Calliclinus geniguttatus (Valenciennes, 1836), C. nudiventris Cervigón \& Pequeño, 1979 y Labrisomus (Labrisomus) philippii (Steindachner, 1866). Esta familia ha sido objeto de diversos cambios taxonómicos por parcialidades, lo cual ha dificultando su visión de conjunto, no sólo por la falta de un documento que unifique estas modificaciones, sino también por la similitud corporal que presentan sus taxa componentes. La presente clave reúne las modificaciones ocurridas y comenta los diferentes cambios taxonómicos y las sinonimias sobre estos peces, desde su descripción hasta la actualidad. Las características diagnósticas han sido seleccionadas, para facilitar el estudio de cada una de las especies que conforman este taxón, permitiendo hacer una diagnosis rápida y precisa de los labrisómidos chilenos.
\end{abstract}

Palabras claves: Peces litorales, reconocimiento, taxonomía, Chile.

\begin{abstract}
A taxonomic key of the Labrisomid fishes from Chile is presented. This taxon is conformed by Auchenionchus crinitus (Jenyns, 1842), A. microcirrhis (Valenciennes, 1836), A. variolosus (Valenciennes, 1836), Calliclinus geniguttatus (Valenciennes, 1836), C. nudiventris Cervigón \& Pequeño, 1979, and Labrisomus (Labrisomus) philippii (Steindachner, 1866). This family has suffered numerous but partial taxonomic changes, making its study difficult not only because of the lack of an unifying document where all these changes are registered, but also because of the high degree of morphological similarity among their component taxa. The present key collected those modifications and comments on diverse taxonomic changes and the synonymies about these fishes, from their description until now. The diagnostic characters have been selected to facilitate the study of each species that represents this taxon, allowing an accurate and fast diagnosis in Chilean labrisomid fishes.
\end{abstract}

KEYwords: Littoral fishes, identification, taxonomy, Chile.

\section{INTRODUCCION}

La familia Labrisomidae de Chile está constituida por Auchenionchus crinitus (Jenyns, 1842), A. microcirrhis (Valenciennes, 1836), A. variolosus (Valenciennes, 1836), Calliclinus geniguttatus
(Valenciennes, 1836), C. nudiventris Cervigón \& Pequeño, 1979, y Labrisomus (Labrisomus) philippii (Steindachner, 1866) (Pequeño 1989, Pequeño \& Lozano 1996). Una séptima especie, Labrisomus fernandezianus (Steindachner, 1866) descrita para la Isla Juan Fernández, es considerada nomen dubium 
ya que se desconoce el tipo (Eschmeyer 2008).

La sistemática y taxonomía de esta familia, ha sufrido notables cambios desde que se consideró a Labrisomidae como un grupo hermano de Clinidae (Hubbs 1952). Por muchos años, diversos autores consideraron a los componentes de Labrisomidae como integrantes de la familia Clinidae (Hubbs 1953, Springer 1959, De Buen 1962, Penrith 1969, Stephens \& Springer 1974). Bajo este supuesto, se consideraban los nombres genéricos Labrisomus (tribu Labrisomini), Calliclinus y Auchenionchus (tribu Cryptotremini) como integrantes de la subfamilia Clininae (Fowler 1945, Steinitz 1950, Yánez 1955, De Buen 1962, Pequeño 1978, Cervigón et al. 1979, Stephens \& Springer 1974). Luego, Springer (1970) tentativamente elevó a Labrisomidae al rango de familia. Se le consideró como un taxa menos especializado que Clinidae y a la vez se dudaba de la relación estrecha que hasta el momento se sostenía entre ambas. Por consecuencia, se redefine Clinidae (George \& Springer 1980) y se excluye de ésta a Tripterygiidae, Labrisomidae y Chaenopsidae y se adiciona la tribu Ophiclinini (Stepien 1992).

Este cambio en la composición taxo - sistemática de la familia Labrisomidae, se asienta posteriormente con estudios en aloenzimas y por secuencias de ADN (Stepien 1992, Stepien et al. 1993). Actualmente aquellos cambios taxo - sistemáticos de Labrisomidae se mantienen, sin embargo la literatura trata estos cambios aisladamente, sin tener aún una herramienta que unifique estas modificaciones y facilite el reconocimiento de estos peces, dificultando aún más el estudio de éstos en su conjunto, debido a la gran similitud morfológica que presentan.

El objetivo de este trabajo es reunir todos los cambios taxonómicos efectuados en los peces de la familia Labrisomidae de Chile en una clave actualizada, con indicación de la distribución geográfica y comentario de las sinonimias de las especies chilenas. De esta manera, se pretende fortalecer los conocimientos taxonómicos, sistemáticos y geográficos sobre dicha familia, lo que facilitará nuevos estudios.

\section{MATERIAL Y METODOS}

Para la elaboración de la clave se revisaron y seleccionaron los caracteres taxonómicos considerados en la literatura (Fowler 1951, Hubbs 1953, De Buen 1962, Stephens \& Springer 1974, Cervigón et al. 1979, Inzunza \& Pequeño 1988, Cárdenas \& Pequeño 1995, Chirichigno \& Vélez 1998).

Las figuras de la clave fueron tomadas y modificadas desde Stephens \& Springer (1974) y Chirichigno \& Vélez (1998).

Abreviaturas de los acrónimos mencionados en el texto (según Leviton et al. 1985): ANSP: Academy of Natural Sciences, Philadelphia, Pennsylvania, U.S.A.; BMNH: British Museum of Natural History; EBM: Universidad de Chile, Estación Biología Marina de Montemar, Universidad de Valparaíso, Chile; IZUA: Instituto de Zoología, Universidad Austral de Chile, Valdivia, Chile; MNHN: Muséum National d'Histoire Naturelle, Paris.

\section{Clave taxonómica de los peces de la familia Labrisomidae de Chile}

1a. Dorsal XIX-XX, 11-13 radios, 68-74 escamas en la línea lateral, radios de la aleta caudal no ramificados: - Pectoral 14-16 (usualmente 15), anal II, 18-20; primera espina aleta dorsal corta, igual a la longitud del opérculo (Norte del Perú hasta Coquimbo, Chile)...............................Labrisomus (Labrisomus) philippii

(Fig. 1)

1b. Dorsal XX- XXVI, 9-15 radios, 40-64 escamas en la línea lateral, radios de la aleta caudal ramificados

2a. Aleta pectoral con 13-14 radios, pélvica I, 3; caudal 13, dos parches nucales 56-64 escamas en la línea lateral. .Auchenionchus. $3 \mathrm{a}$

2b. Aleta pectoral con 14-15 radios, pélvica I, 4; caudal 13, tres parches nucales (excepcionalmente dos), 40-48 escamas en la línea lateral. Calliclinus...5a 
3a. Aleta dorsal XXIV-XXV; 11-12, pectoral 14, anal II, 22. Escamación incompleta (especimenes sobre los $174 \mathrm{~mm}$ de LS); sin escamas en las membranas de las aletas; peritoneo negro (Iquique hasta Río Lar, en la costa abierta de Isla Chiloé, Chile).

A. variolosus

(Fig. 2)

3b. Aleta dorsal XXV-XXVI; 9-12, pectoral 13, anal II, 23-24. Escamas cubren la totalidad del cuerpo y bases de las aletas, extendiéndose hacia las membranas (especimenes sobre $50 \mathrm{~mm}$ de LS); peritoneo blanco..... $4 \mathrm{a}$

4a. Número total de cirros nucales y orbitales 20-45, menos de 18 dientes en cada palatino (Pucasana, Perú hasta Viña del Mar, Chile). A. crinitus

(Fig. 3)

4b. Número total de cirros nucales y orbitales menos de 20, numerosos dientes en cada palatino (50-100) (Isla Vieja, Perú hasta Valparaíso, Chile). A. microcirrhis

5a. Vientre y axilas pectorales con escamas, dorsal XXIV-XXVI, 11-13; pélvica I, 3-4; anal 21-22. Ausencia de bandas oscuras en la región ventral de la mandíbula. Color pardo verdoso con áreas alternadas claras y oscuras sin formar bandas bien definidas. Por debajo de la dorsal cinco manchas rojizas o rosadas espaciadas entre sí. Otra mancha rojiza en el extremo superior del opérculo (Desde Antofagasta hasta Punta Arenas, Chile)..... C. geniguttatus

(Fig. 5 a)

5b. Vientre y axilas pectorales sin escamas, dorsal XXII-XXIII, 11-13; pélvica I, 3; anal 19-21. Presencia de bandas oscuras en la región ventral de la mandíbula. Franjas verticales pardo oscuro y claras alternadas, pero no bien definidas ni regularmente dispuestas (Caleta Leandro hasta costa interior de Quellón y sur de la Isla de Chiloé).

C. nudiventris

(Fig. 5 b)

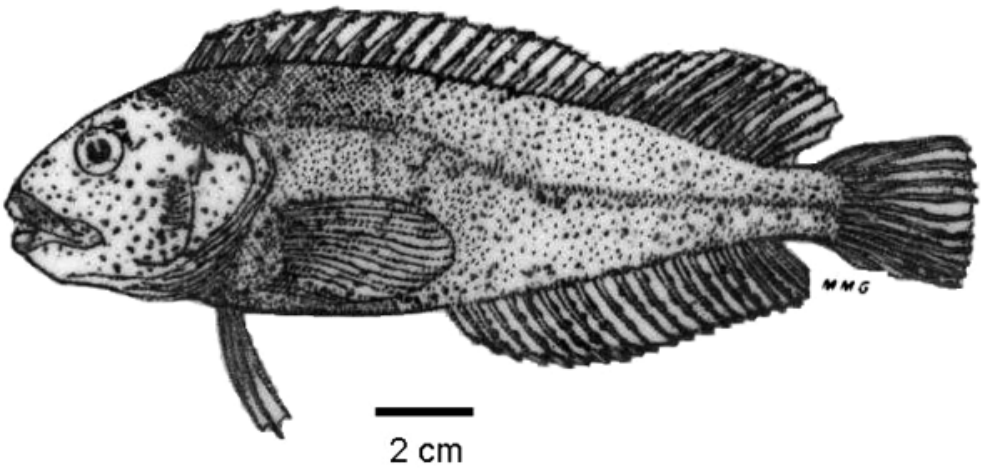

FIGURA 1. Labrisomus (Labrisomus) philippii (Steindachner, 1866) (Modificado de Chirichigno \& Vélez 1998).

Figure 1. Labrisomus (Labrisomus) philippii (Steindachner, 1866) (Modified from Chirichigno \& Vélez 1998). 


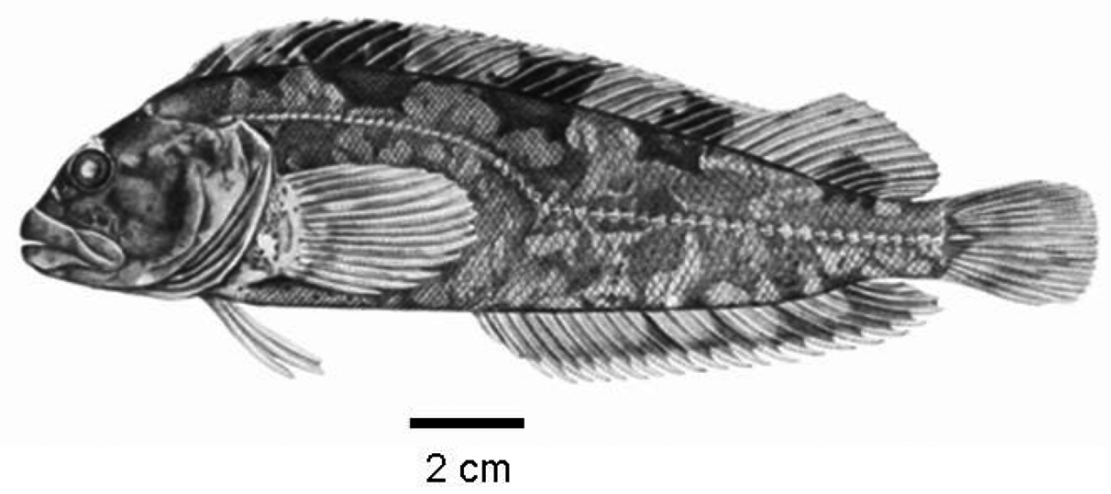

FIGURA 2. Auchenionchus variolosus (Valenciennes, 1836) (Modificado de Stephens \& Springer 1974).

FIGURE 2. Auchenionchus variolosus (Valenciennes, 1836) (Modified from Stephens \& Springer 1974).

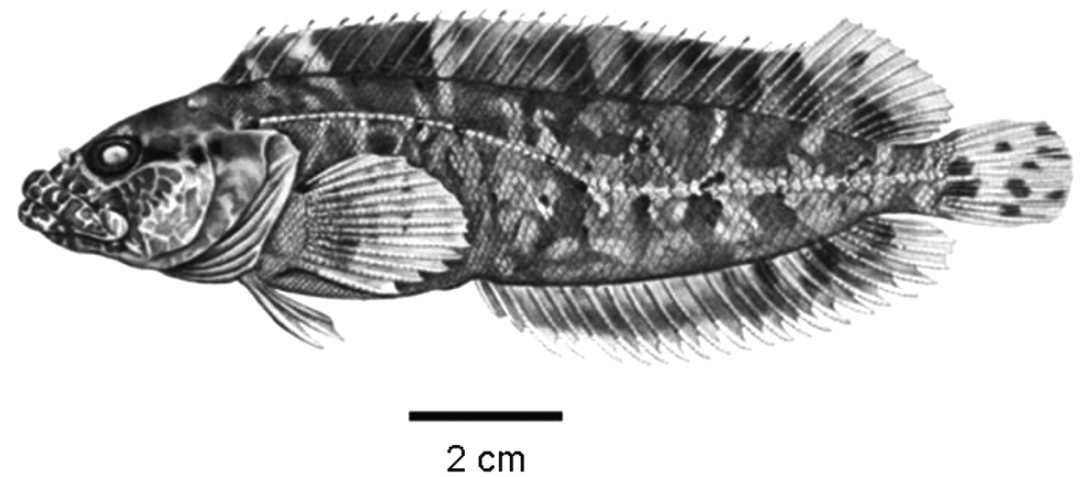

FIGURA 3. Auchenionchus crinitus (Jenyns, 1842) (Modificado de Stephens \& Springer 1974).

FIGURE 3. Auchenionchus crinitus (Jenyns, 1842) (Modified from Stephens \& Springer 1974). 
Gayana 73(1), 2009

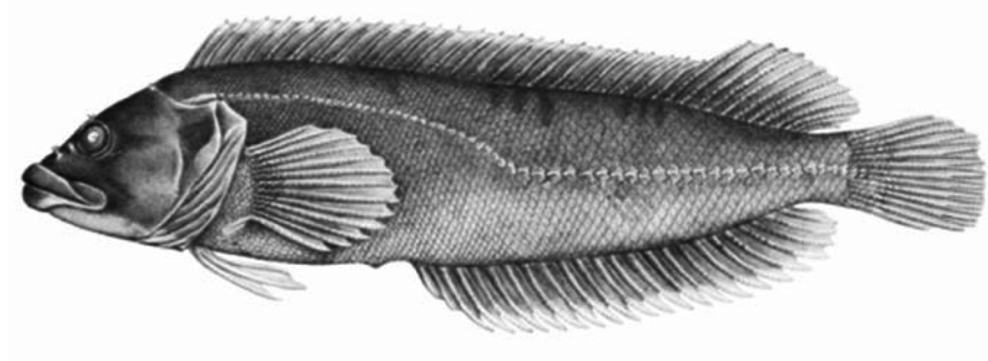

$2 \mathrm{~cm}$

FIGURA 4. Auchenionchus microcirrhis (Valenciennes, 1836) (Modificado de Stephens \& Springer 1974).

FIGURE 4. Auchenionchus microcirrhis (Valenciennes, 1836) (Modified from Stephens \& Springer 1974).
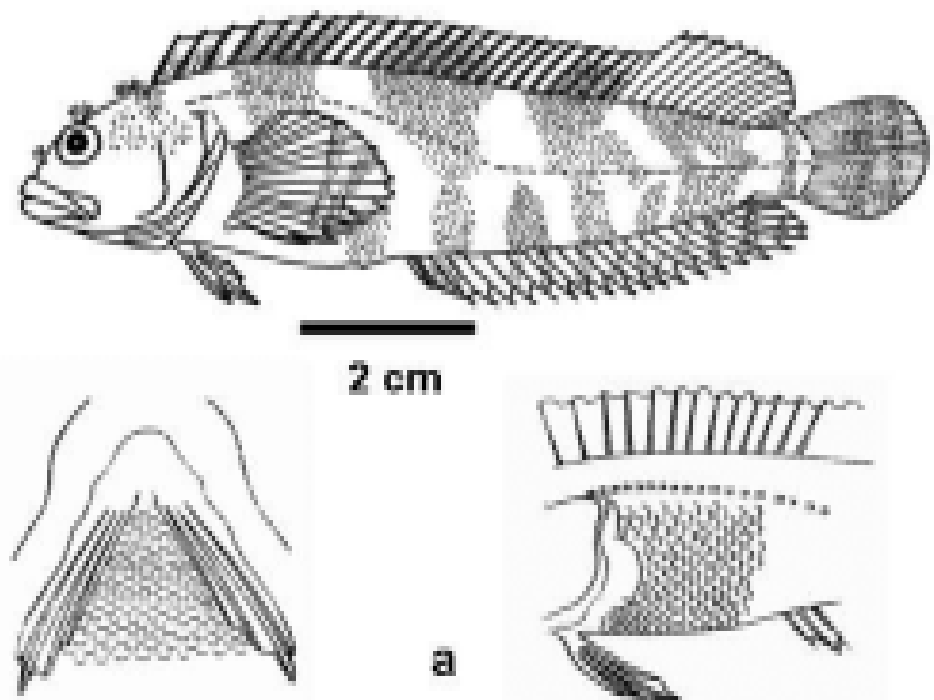

$2 \mathrm{~cm}$

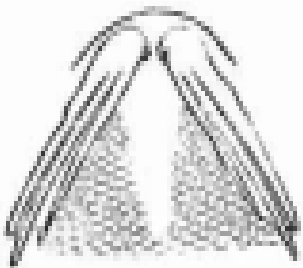

a
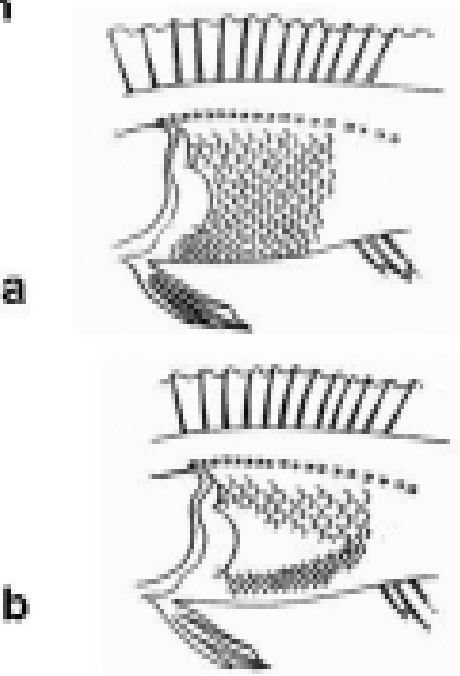

Figura 5. Esquema de las principales diferencias taxonómicas entre las especies de Callilcinus: a) C. genigutattus (Valenciennes, 1836). b) C. nudiventris Cervigón \& Pequeño, 1979 (Modificado de Pequeño \& Sáez 2004).

Figure 5. Scheme of the main taxonomic differences between Calliclinus species: a) C. genigutattus (Valenciennes, 1836). b) C. nudiventris Cervigón \& Pequeño, 1979 (Modified from Pequeño \& Sáez 2004). 


\section{Comentarios sobre los géneros y especies de la familia Labrisomidae de Chile}

Auchenionchus Gill, 1860

Auchenionchus Gill, 1860:103 (originalmente dado como Anchenionchus; primera corrección Abbot, 1899; ortografía original considerada errónea por Jordan, 1919; especie tipo: Clinus variolosus Valenciennes, designación original).

Flabelliclinus De Buen, 1962:57 (propuesto como un género de Auchenionchus; especie tipo: Auchenionchus verrucosus De Buen, 1962 (= Clinus microcirrhis Valenciennes), por designación original).

Chalacoclinus De Buen, 1969:57 (propuesto como un subgénero de Auchenionchus; especie tipo: Auchenionchus chalaco De Buen, 1962 (= Clinus crinitus Jenyns), por designación original).

Género reconocido válido sólo por Abbott en 1899 y por Fowler en 1944 desde su descripción, haciéndose una distinción con Labrisomus por algunos caracteres morfológicos tales como: radios caudales ramificados y por los cortos y simples cirros nucales (Hubbs 1952).

Los representantes de este género se agrupan en tres especies que habitan las costas del Pacífico suroriental: A. crinitus, A. microcirrhis, A. variolosus se encuentran en simpatría en la zona de Valparaíso (Stephens \& Springer 1974, Cárdenas \& Pequeño 1996). En general, son peces con características muy similares entre ellos y con una biología prácticamente desconocida.

a) A. crinitus (Jenyns, 1842):

Clinus crinitus Jenyns, 1842:90 (Coquimbo, Chile; holotipo, BMNH 1917.714.55, Beagle).

Auchenionchus chalaco De Buen, 1962:70 (Antofagasta; holotipo, EBM 10.022, aparentemente perdido).

A. crinitus fue también descrita como Auchenionchus (Chalacoclinus) chalaco por De Buen (1962), luego dicho autor separa a esta última de otros miembros del género debido a su reducido número de elementos en la aleta pélvica. Posteriormente Stephens \& Springer (1974) revisan el holotipo notando que las aletas pélvicas son cortas y malformadas, lo cual indicaría que se trataba de un especímen aberrante y que la falta de elementos de la aleta pélvica era producto de esta malformación. Morfológicamente, los adultos de Auchenoinchus crinitus son fácilmente distinguibles de otras especies del mismo género, como microcirrhis por el número de dientes palatinos y el número de cirros en la cabeza (Stephens \& Springer 1974).

La situación actual de la especie es que se acepta como válido el nombre binomial Auchenionchus crinitus (Stephens \& Springer 1974, Cárdenas \& Pequeño 1995, Pequeño 1989, Eschmeyer 2008).

b) A. microcirrhis (Valenciennes, 1836)

Clinus microcirrhis Valenciennes en Cuvier y Valenciennes, 1836:384 (costas de Valparaíso; sintipo, MNHN, A. 2072, A.2126).

Clinus peruvianus Valenciennes, 1836:383 (sin tipo o localidad tipo designada; probablemente Perú).

Clinus niger Philippi, 1896:377 (Chile; holotipo aparentemente destruido).

Auchenionchus tentaculatus De Buen, 1962:63 (Arica; holotipo, EBM 10.356, aparentemente destruido).

Auchenionchus verrucosus De Buen, 1962:68 (Antofagasta; holotipo, EBM 10.021, aparentemente destruido).

Auchenionchus miniatus De Buen, 1962:60 (Valparaíso; holotipo, EBM 10.372, aparentemente destruido)

Otra especie conocida en Chile, es A. microcirrhis, cuya historia taxo - sistemática ha sido algo compleja debido a que las descripciones originales se referían al género Clinus (1836 - 1896) pasando por distintas especies. Luego, De Buen (1962) cambia el género a Aucheniounchus. El mismo De Buen, realizó cambios intragenéricos nominales al crear tentaculatus, verrucosus y miniatus. Los estudios realizados por dicho autor, se refieren a diferenciar a A. miniatus de A. microcirrhis basándose sólo en el color y con un número desigual de ejemplares (uno de miniatus y cuatro de microcirrhis). Posteriormente, Stephens \& Springer (1974) revisaron la descripción de miniatus concordando con el ejemplar de microcirrhis, lo que llevó a estos autores a considerarlos como sinónimos.

Cabe destacar que Stepien (1990) sugiere que $A$. microcirrhis y A. crinitus son una sola especie y podrían ser sinónimos quedando sólo $A$. microcirrhis. Sin embargo, en la actualidad queda descartado lo propuesto por esta autora ya que ambas especies siguen consideradas como válidas (Eschmeyer 2008).

Por su parte, tentaculatus fue separada por De Buen de microcirrhis por sus grandes ojos. Sin embargo, lo que no consideró De Buen, fue que el diámetro 
ocular va variando con el tamaño, por lo cual Stephen \& Springer (op. cit.), lo consideran a tentaculatus como sinónimo de microcirrhis. Por su parte, A. verrucosus fue descrita a partir de un único ejemplar, el cual poseía un número reducido de cirros orbitales y nucales lo que indicaba que esta especie podría ser incluida como un sinónimo de A. microcirrhis. Actualmente sólo se reconoce como válida a esta última especie (Pequeño 1989; Eschmeyer 2008).

c) A. variolosus (Valenciennes, 1836)

Clinus variolosus Valenciennes en Cuvier \& Valenciennes 1836 (Valparaíso, Chile; holotipo, MNHN A. 2170).

Calliclinus validus De Buen, 1962:80 (Valparaíso; holotipo EBM, aparentemente destruido).

A. variolosus fue genéricamente considerada como Calliclinus validus por De Buen (1962), basándose en el número de cirros nucales y orbitales. Stephens \& Springer (1974), analizan tales caracteres en ejemplares de $A$. variolosus dando cuenta que éstos varían, y que los cirros orbitales a pesar que siempre son divididos, varían en su grado de ramificación. La nueva especie descrita por De Buen, C. validus, se basó sobre un solo ejemplar coincidiendo con el holotipo de $C$. variolosus, excepto que tenía sólo cuatro branquispinas en el brazo inferior del primer arco branquial. Los cinco especímenes examinados por Stephens \& Springer (op. cit.) incluyendo el holotipo tenían menos de siete branquispinas, siendo dos o tres de ellas rudimentarias. De esta manera se considera como válida la especie actualmente conocida como Auchenionchus variolosus (Valenciennes, 1836) (Pequeño 1989, Eschmeyer 2008).

\section{Aspectos biogeográficos de las especies del género Auchenionchus:}

Geográficamente, cabe destacar que la distribución que actualmente se conoce en este taxón ha presentado algunos cambios desde las descripciones originales. De este modo, las tres especies reconocidas aparecieron juntas en La Ventana (Chile) cerca de Valparaíso. A. crinitus fue descrita por primera vez para Coquimbo $\left(30^{\circ} \mathrm{S}\right)$ y luego para Iquique $\left(20^{\circ} \mathrm{S}\right)$. A. microcirrhis, fue originalmente descrita para Valparaíso y posteriormente se conoció en Bahía Independencia, Perú (Stepien 1990). Finalmente $A$. variolosus, fue descrita originalmente para Valparaíso y luego se amplió su distribución desde Iquique al extremo austral de Chile (Mann
1954). Después, fue conocida también en Tomé (36³8'S) (Stephens \& Springer 1974), pero hacia el sur solo hay evidencia de su presencia hasta la costa del norte de la isla grande de Chiloé (Cárdenas $\&$ Pequeño 1995). Pensamos que A. variolosus se distribuyó secundariamente al sur de Valparaíso mientras que $A$. microcirrhis y A. crinitus están distribuidas más hacia el norte, con una distribución septentrional más antigua

Cabe destacar que el género Auchenionchus se ha encontrado más hacia el sur de lo conocido originalmente y, en el caso de A. variolosus que logra llegar hasta Chiloé, hace pensar que sus capacidades de dispersión se ven frenadas por factores tanto bióticos (temperatura) como por la presencia de una diversidad de peces litorales inter y submareales que no existen o disminuyen en número de especies desde Chiloé hacia el norte. A estos factores se le suman las barreras zoogeográficas que influyen en la distribución de otros organismos litorales (Cárdenás \& Pequeño 1995). Un aspecto importante de señalar es que a diferencia de lo señalado por Stepien (1990.). A. variolosus no es una especie que sólo permanezca en la zona intermareal, sino también se encuentra en la zona submareal (Cárdenas \& Pequeño op. cit.)

Calliclinus Gill, 1860

Calliclinus Gill, 1860:103 (especie tipo: Clinus geniguttatus Valenciennes, por designación original).

Myersiccthys Hubbs, 1952:103 (especie tipo: Clinus guttulatus Valenciennes (=Clinus geniguttatus), por designación original).

Pennaclinus De Buen, 1962:82 (especies tipo: Pennaclinus racemarius De Buen (= Clinus guttulatus), por designación original).

Género creado por Gill en 1860, reconocido por Fowler en 1944 y relacionado con la familia Clinidae (Hubbs 1952, De Buen 1962, Cervigón et. al. 1979, Inzunza \& Pequeño 1988). Hasta entonces, Clinidae representaba a cuatro géneros: Calliclinus, Myxodes, Auchenionchus y Labrisomus (Hubbs 1952, Pequeño 1978). Actualmente Calliclinus es considerado como integrante de la familia Labrisomidae (Pequeño 1989, Pequeño \& Lozano1993, Eschmeyer 2008).

El actual género Calliclinus llegó a estar compuesto por varios géneros, hoy considerados sinónimos (Calliclinus, Myersichthys y Pennaclinus). Además, este género estuvo muy vinculado con Auchenionchus, 
puesto que Calliclinus poseía sinonimia con éste, por un error de identificación (Hubbs 1952). Luego, aparecieron las otras sinonimias del género (Stephens \& Springer 1974). Actualmente, es válido como Calliclinus (Eschmeyer 1998) y está compuesto por dos especies habitantes de sectores intermareales y sublitorales: $C$. geniguttatus y $C$. nudiventris, las cuales viven en simpatría parcial (Cervigón et al. 1979, Inzunza \& Pequeño 1988).

a) C. geniguttatus (Valenciennes, 1836)

Clinus geniguttatus Valenciennes en Cuvier y Valenciennes 1836:386 (Valparaíso, tres sintipos, MNHN A. 2069).

Clinus elegans Valenciennes en Cuvier y Valenciennes 1836:386 (Valparaíso, dos sintipos, MNHN A. 2073).

Clinus guttulatus Valenciennes en Cuvier y Valenciennes 1836:387 (Valparaíso, tipo perdido; lectotipo de Clinus genigutatus, MNHN A. 2069, designado como neotipo de C. guttulatus en Stephens \& Springer 1974).

Clinus guttatus Guichenot en Gay 1848:248.

Labrisomus conventryi Fowler, 1940:187 (Mocha, Chile; holotipo ANSP 69149).

Pennaclinus racemarius De Buen, 1962:82 (Chan Chan en Valdivia, tipo probablemente destruido).

Esta especie ha tenido variados cambios taxonómicos relativamente complejos. El primer sinónimo de la especie fue C. elegans por Steindachner (1898). Posteriormente, Valenciennes distinguió a esta especie por el color de uno de los sintipos. Luego, utilizando datos merísticos de la especie se provocaron las posteriores sinonimias. Actualmente es válido el binomio Calliclinus geniguttatus (Valenciennes, 1836) (Eschmeyer 2008).

b) C. nudiventris Cervigón \& Pequeño, 1979

Calliclinus nudiventris Cervigón y Pequeño: 40 (Chile; holotipo, Linao Isla de Chiloé IZUA PM, 266). Este ejemplar fue revisado durante la preparación del manuscrito, pero el día 3 de diciembre, 2007, fue destruido por un incendio.

Especie de creación relativamente nueva. Afortunadamente, no ha tenido las complicaciones taxonómicas que su antecesora. Se distingue de geniguttatus principalmente por la presencia de un área triangular alargada (Fig. 5b), desprovista de escamas en la región ventral, situada por detrás de las aletas pélvicas; presencia de un área en la región axilar postpectoral inferior sin escamas (Cervigón
\& Pequeño 1979). Internamente, el análisis osteológico ha revelado también que existen diferencias en el maxilar, premaxilar y el dentario de ambas especies, presentando geniguttatus una mayor protractibilidad bucal que nudiventris (Inzunza \& Pequeño 1988).

El nombre binomial C. nudiventris, es actualmente válido y no ha sufrido cambios taxonómicos (Eschmeyer 2008).

\section{Aspectos geográficos de las especies del género Calliclinus:}

Geográficamente, las especies poseen diferencias en su rango de distribución. Mientras geniguttatus se extiende desde Antofagasta hasta Punta Arenas, el rango de distribución de nudiventris es menor extendiéndose desde Caleta Leandro (Concepción) hasta Quellón (Chiloé) (Cervigón \& Pequeño 1979). $C$. nudiventris es entonces simpática con $C$. genigttatus en el $100 \%$ de su rango de distribución, pero lo contrario no ocurre.

Labrisomus Swainson, 1839

Labrisomus Swainson, 1839:277 (tipo, Clinus pectinifer Cuvier \& Valenciennes por designación original).

Lepisoma De Kay, 1842:41 (tipo Lepisoma cirrhosum De Kay por monotipia).

Labrosomus Gill, 1860:105 (sustituído por Labrisomus Swainson, mismo tipo).

Ericteis Jordan, 1904:543 (tipo Ericteis kalisherae Jordan por designación original).

Odontoclinus Reid, 1935:164 (tipo Odontoclinus dendriticus Reid por designación original).

El género Labrisomus fue propuesto por Swainson en 1839, el cual incluía 12 especies todas creadas por Cuvier \& Valenciennes y se designaba a Clinus pectinifer como especie tipo (Hubbs 1953).

Springer (1958) consideró a Labrisomus philippii como el integrante más primitivo de los géneros, basándose principalmente en el gran tamaño corporal, aumento en la dentición, características de la línea lateral y la merística de la aleta pectoral. A la vez advierte que la especialización dentro del género Labrisomus involucra la disminución del tamaño corporal, dentición y escamación.

Dos son los integrantes de este género descritas para Chile: L. (Labrisomus) philippii y L. fernandezianus. Esta última se mantiene como nom. $d u b$., ya que se 
desconoce el tipo y no se conoce registro de captura desde su descripción original en la Isla Juan Fernández (Fowler 1943). Por esta razón, esta especie se excluye de la presente clave.

Menos complejo es el caso de L. (Labrisomus) philippii (Steindachner, 1866), sin embargo, no ha estado ajeno a algunas sinonimias:

Clinus philippii Steindachner, 1866:210 (costa oeste de Sur América; holotipo presumiblemente en el Museo Naturshistorisches de Viena).

Clinus fortidentatus Cope, 1877:42 (Bahía del Callao; holotipo aparentemente perdido).

Clinus chilensis Sauvage, 1883:157 (Chile: holotipo, MNHN A. 4870).

Actualmente es válido como L. (Labrisomus) philippii (Eschmeyer 2008).

Aspectos geográficos de L. (Labrisomus) philippii: Su distribución geográfica es más amplia que fernandezianus (de existir ésta última), extendiéndose desde Perú hasta Coquimbo (Hubbs 1953, Stephens \& Springer 1974).

\section{DISCUSION Y CONCLUSIONES}

A pesar de tratarse de un grupo pequeño de especies, la complejidad sistemática y taxonómica de los integrantes de la familia Labrisomidae, se manifiesta al analizar su historia taxo - sistemática y su distribución geográfica, respecto a Clinidae. Esta última familia es considerada como un taxón altamente especializado, y se distribuye alrededor de aquellos considerados más primitivos como Labrisomidae (Springer 1970). Además los labrisómidos están más restringidos a los trópicos del Nuevo Mundo, con sólo cuatro especies derivadas que aparecen con amplia distribución (Spriger $o p$. cit.) y, en muchos aspectos es menos especializado que Clinidae, especialmente en el tipo de escamas, la falta de un proceso (como gancho) en el cleitrum, la ocasional presencia de radios caudales ramificados y la dentición no reducida en muchos géneros. Sin embargo, en otros caracteres ellos parecen ser más especializados como en el gran desarrollo de los cirros de la cabeza. Otro aspecto importante de destacar es el tipo de reproducción que presentan ambas familias; en la mayoría de las especies de Clinidae se presenta la viviparía, donde los machos poseen un órgano copulador (con testículo impar) mientras que en
Labrisomidae sólo el género Starksia Jordan \& Evermann 1896 es vivíparo, y el órgano copulador es diferente al que presenta Clinidae, de esta manera Starksia parece ser una rama especializada de Labrisomidae (posee testículos pares) más que un intermedio entre las dos familias o derivado de Clinidae (Springer 1970; Rasotto 1995). Cabe mencionar que uno de los autores (G. Pequeño) ha visto desovar una hembra de Myxodes (Clinidae), probablemente $M$. viridis, en el laboratorio, contando un racimo de más de 60 huevos.

Así, se ha podido discriminar la situación de Clinidae respecto a Labrisomidae que por tantos años eran consideradas como una sola familia (Hubbs 1953, Springer 1959, De Buen 1962, Penrith 1969, Stephens \& Springer 1974). Los análisis del ADN de los peces de ambas familias han dado como resultado que a pesar de ser familias muy emparentadas, las especies integrantes de cada una de ellas poseen diferencias genéticas, las cuales se reflejan en los fenotipos, por tenues que sean y por eso ameritan una separación. Hoy en día se sabe que los análisis más parsimoniosos en la secuencia de $\mathrm{ADN}$, indican que Labrisomidae es parafilético, resolviendo en parte la relación con Clinidae (Stepien et al. 1993). Sin duda que aquello es un gran avance en la sistemática y taxonomía de ambas familias.

Nuestra clave, apuntó a reunir estos cambios, entregando así la información taxonómica aceptada en la actualidad (Stepien 1992, Stepien et al. 1993, Chirichigno \& Vélez 1998, Eschmeyer 2008).

En general, el reconocimiento de las especies de labrisómidos está dado por una combinación de caracteres que son claves en la determinación específica: número de espinas y radios en la aleta dorsal, pélvica, anal, radios en las aletas pectoral y caudal, escamas de la línea lateral, forma y número de los cirros bucales, número de vértebra y color del peritoneo. Evidentemente que los análisis osteológicos son claves para evidenciar diferencias; alguna información existe referente a $A$. crinitus y los integrantes de Calliclinus (Cárdenas \& Pequeño 1995, Inzunza \& Pequeño 1988). En este trabajo, se escogieron aquellos caracteres diagnósticos, que facilitan la observación tanto externa como interna de los especímenes, dando una herramienta de apoyo práctico para el investigador, dada la similitud morfológica de los labrisómidos chilenos.

Otro aspecto importante, es la distribución geográfica de las especies chilenas de Labrisomidae. Cada uno de los taxa posee características 
fisiológicas que aparentemente están relacionados con tan variada distribución. Dos de las especies de Auchenionchus muestran un patrón geográfico marcadamente más septentrional (A. crinitus y $A$. microcirrhis), sin embargo, al parecer ciertos factores bióticos y abióticos impiden a estas especies avanzar más hacia el sur, como lo hace $A$. variolosus que a diferencia de las otras dos puede desplazarse hacia aguas más frías (Cárdenas \& Pequeño 1995). Algo similar ocurre con las especies del género Calliclinus, donde C. geniguttatus posee la capacidad de avanzar desde aguas del norte de Chile hacia zonas más frías, mientras que $C$. nudiventris se restringe a ambientes climáticamente más intermedios, con respecto a $C$. geniguttatus.

No escapa a estas observaciones Labrisomus. Con una especie que también vive en el Perú y llega hasta Coquimbo como L. (Labrisomus) philipii (Stephens \& Springer 1974), comparado con $L$. fernandezianus, del cual sólo se tienen datos de haber sido capturado en la Isla Juan Fernández y que hasta hoy no se conoce captura alguna desde su descripción original (Fowler 1945, Eschmeyer 2008). Creemos que, muy probablemente, $L$. fernandezianus sea sinónimo de alguna de las especies litorales de ese archipiélago, como podría ser el caso de algún lábrido (Familia Labridae).

De esta manera, se puede apreciar un avance en la situación taxonómica de Labrisomidae, sin embargo, aún queda mucho por estudiar en cuanto a otros aspectos biológicos de las especies, que en la literatura son abordados de manera somera e incompleta. Algunos caracteres taxonómicos tan útiles y discriminantes como son las estructuras de la línea lateral (canales y poros cefálicos), serían de gran apoyo para distinguir y conocer mejor la taxonomía y biología de estos peces. Si se desea entender los patrones de distribución geográfica de las especies, así como las diferencias morfológicas (externas e internas), se debe crear conciencia entre los ictiólogos, acerca que estos aspectos son importantes en el estudio de la familia para tener una visión integral de las especies constituyentes del taxón, tarea que quedará como desafío para el futuro.

\section{AGRADECIMIENTOS}

Se agradece el valioso aporte bibliográfico del Dr. Víctor G. Springer (Smithsonian Institution, Washington, D. C.). Don León Matamala (Instituto de Zoología, Universidad Austral de Chile) prestó apoyo en el laboratorio.

Estos son resultados parciales del Proyecto DIDUACH No 2005-03.

\section{BIBLIOGRAFIA}

Cardenas, V. \& G. Pequeño. 1995. Nuevo registro de Auchenionchus variolosus (Valenciennes, 1836) y estudio alimentario preliminar (Osteichthyes, Labrisomidae). Ciencia y Tecnología del Mar 18: 3-12.

Cervigón, F., G. Pequeño \& C. Moreno. 1979. Descripción de Calliclinus nudiventris nov. sp. y notas adicionales sobre $C$. geniguttatus (Pisces, Clinidae). Medio Ambiente 4(1): 40 - 50.

Chirichigno, N. \& J. Velez. 1998. Clave para identificar los peces marinos del Perú (Segunda edición revisada y actualizada). Publicación Especial Instituto del Mar del Perú, 302 pp.

De Buen, F. 1962. Fauna Chilena. Peces de la Familia Clinidae. Montemar 2: 53-90. (Parte de la Revista Biología Marina, Valparaíso).

Eschmeyer, W. N. 2008. Catalog of Fishes. Cal. Acad. Sci., San Francisco.

(Accesado: Agosto, 2008). http://www. calacademy.org

Fowler, H. 1945. Fishes of Chile. Systematic Catalog Reprint. Revista Chilena de Historia Natural Parts I y II, 36:171 pp., Santiago.

Fowler, H. 1951. Analysis of the fishes of Chile. Revista Chilena de Historia Natural 51-53: 263- 326.

George, A. \& V. Springer. 1980. Revision of the clinid fish tribe Ophiclinini, including five new species, and definition the family Clinidae. Smithsonian Contributions to Zoology 307: 1-30.

HubBs, C. 1952. A contribution to the classification of the blenniod fishes of the Familiy Clinidae, with partial revision of the Eastern Pacific forms. Stanford Ichthyology Bulletin 4(2): 41-157.

HubBs, C. 1953. Revision of the Eastern Pacific fishes of the clinid fish genus Labrisomus. Zoologica, New York 38 (3): 113-136.

InZunza, A. \& G. PequeÑo. 1988. Aspectos trófico adaptativos y reafirmación de dos especies en el género Calliclinus Gill 1860 (Osteichthyes, Clinidae). Boletín de la Sociedad Biológica de Concepción 59: 69-93.

MANN, G. 1954. La vida de los peces en aguas chilenas. Instituto de Investigaciones Veterinarias y Universidad de Chile, 343 pp., Santiago.

Penrith, M. L. 1969. The systematics of the fishes of the family Clinidae in South Africa. Annals of the South African Museum 55(1): 1-121.

Pequeño, G. 1978. Doce nuevos registros de peces para la costa de Valdivia, Chile, y su alcance ictiogeográfico. Revista Comisión Permanente Pacífico Sur 9: 109-126.

Pequeño, G. 1989. Peces de Chile. Lista sistemática 
Gayana 73(1), 2009

revisada y comentada. Revista Biología Marina, Valparaíso 24(2): 1-132.

Pequeño, G. \& X. Lozano. 1996. Las familias del Suborden Blenniodei de Chile, con una recopilación bibliográfica del área. Documentos Técnicos en Zoología, Instituto de Zoología, Univ. Austral de Chile 2: 13.

RasotTo, M. 1995. Male reproductive apparatus of some Blennioidei (Pisces: Teleostei). Copeia 1995(4): 907 - 914.

SPRINGER, V. G. 1959. Systematics and Zoogeography of the Clinid fishes of the Subtribe Labrisomin Hubbs. Institute of Marine Science, University of Texas 5: 417-492.

Springer, V. G. 1970. The Western Atlantic clinid fish Ribeiroclinus eigenmanni with discussion of the interrelationships and zoogeography of the Clinidae. Copeia 3: 430-436.
STEINITZ, H. 1950. On the Zoogeograohy of the Teleostean genera Salarias, Ophioblennius and Labrisomus. Estratto dall ' Archivio Zoologico Italiano 35: 325348

Stephens, J. S. \& V. G. Springer. 1974. Clinid fishes of Chile and Peru, with description of a new species, Myxodes ornatus, from Chile. Smithsonian Contributions to Zoology 159: 1-24.

Stepien, C. 1992. Evolution and biogeography of the Clinidae (Teleostei: Blenniodei). Copeia 2: 375392

Stepien, C., M. Dixon \& D. Hillis. 1993. Evolutionary relationships of the Blennioid fish families Clinidae, Labrisomidae and Chaenopsidae: Congruence between DNA sequence and allozime data. Bulletin of Marine Science 52(1): 496-515.

YAÑEZ, P. 1955. Peces útiles de la costa de Chile. Revista Biología Marina, Valparaíso 6: 29-81.

Recibido: 15.11 .08

Aceptado: 12.05.09 